\title{
Non fatal pulmonary haemorrhage associated with nitrofurantoin
}

\author{
C E BUCKNALL, M R ADAMSON, S W BANHAM \\ From the Departments of Respiratory Medicine and Pathology, Glasgow Royal Infirmary, Glasgow
}

\begin{abstract}
Various adverse pulmonary reactions have been recorded after administration of nitrofurantoin. ${ }^{12}$ Acute pulmonary infiltrates, resolving quickly with withdrawal of the drug, pleural effusions, and (less commonly) a chronic form of pulmonary fibrosis are all described. Fatal pulmonary haemorrhage was recorded by Auerbuch ${ }^{3}$ in an alcoholic patient with a generalised bleeding disorder and postmortem evidence of cirrhosis, but there are no reports of alveolar haemorrhage as part of the acute nitrofurantoin reaction in previously well individuals. We report such a case.
\end{abstract}

\section{Case report}

A previously well 43 year old woman was admitted with a four day history of increasing breathlessness and haemoptysis. Six days before admission she had been treated with nitrofurantoin for a presumed urinary tract infection, after developing loin pain, dysuria, and lower abdominal pain. She had received nitrofurantoin six months earlier with no ill effects, but she had a history of rashes after various drugs, including penicillin. She had a past history of eczema, which was quiescent at the time. She was a smoker.

Physical examination initially showed a breathless and anxious woman who was pale, febrile, and cyanosed. Widespread crackles were audible on auscultation, and her blood pressure was $170 / 90 \mathrm{~mm} \mathrm{Hg}$; no other abnormalities were found. A chest radiograph (fig 1) showed an alveolar filling pattern with normal heart size, suggesting adult respiratory distress syndrome. Blood gas tensions showed type I respiratory failure with an arterial oxygen tension of $28 \mathrm{~mm} \mathrm{Hg}$ $(3.73 \mathrm{kPa})$. The haemoglobin concentration was $7.7 \mathrm{~g} / \mathrm{dl}$, with a neutrophil leucocytosis and no eosinophilia. Urea and electrolye concentrations and the results of liver function tests were normal; urine analysis showed ++ protein and microscopy gave an Addis count of 4 red blood cells, no pus cells, and 2 small granular casts. The results of coagulation studies were entirely normal.

Nitrofurantoin treatment was stopped and she was treated with high flow oxygen. She deteriorated and required positive pressure ventilation for eight days. In view of the severity of her condition, intravenous methyl prednisolone $1 \mathrm{~g}$ on alternate days and cyclophosphamide $180 \mathrm{mg}$ daily were given and plasmapheresis was performed on four occasions. While being ventilated she developed a small right sided pneumothorax, which did not require drainage. Her chest radiograph showed gradual clearing of the interstitial

Address for reprint requests: Dr CE Bucknall, Department of Respiratory Medicine, Glasgow Royal Infirmary, Glasgow.

Accepted 28 November 1986

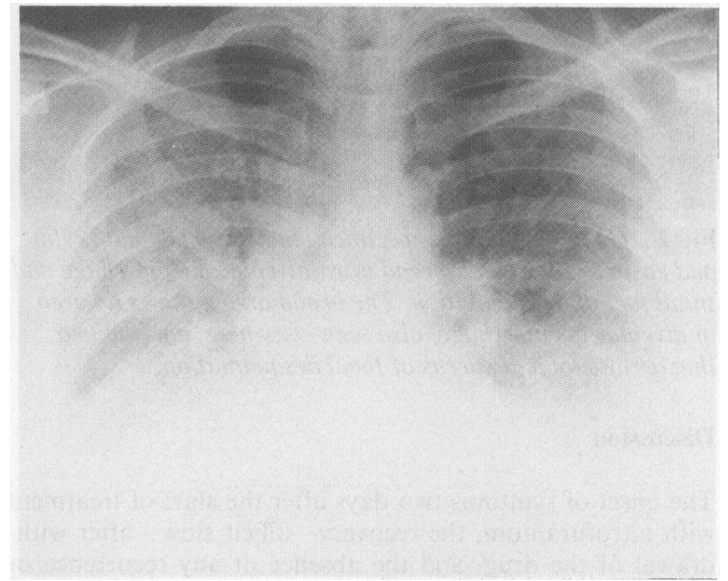

Fig 1 Chest radiograph showing bilateral alveolar infiltrates with normal heart size (adult respiratory distress syndrome).

changes, but her recovery was punctuated by the development of a small blood stained pleural effusion. This settled spontaneously and was not associated with any perfusion abnormalities on ventilation-perfusion scanning. She was discharged on the 27th hospital day and she has had no further respiratory or renal problems over the subsequent year.

When the acute illness failed to improve rapidly on withdrawal of nitrofurantoin, an open lung biopsy was performed on the second hospital day. This showed pronounced alveolar haemorrhage with focal desquamation but no evidence of vasculitis (fig 2). Renal function remained normal throughout and haematuria was never noted. The serum level of antiglomerular basement membrane antibody was $7 \cdot 3 \%$ (normal range $<12 \%$ ) and bacterial and viral cultures of lung tissue were sterile. Pulmonary function testing on the 14th hospital day showed a restrictive ventilatory defect, with vital capacity (VC) 1.75 litre (54\% predicted), $\mathrm{FEV}_{1} / \mathrm{VC}$ ratio $80 \%$, and transfer coefficient (KCO) $2 \cdot 3$ (normal range 1.09-3.05 mmol min ${ }^{-1} \mathrm{kPa}^{-1} \mathrm{l}^{-1}$ ). Subsequent chest radiographs and pulmonary function tests have shown a gradual but complete recovery to normal, the latest VC being 2.651 (83\% predicted), total lung capacity 3.981 ( $94 \%$ predicted), and $\mathrm{KCO} 1 \cdot 8$. The results of a nutritional screen on the 11 th hospital day, at the time the patient was being weaned from ventilation, included normal vitamin $\mathrm{E}$ concentrations; a dietary history revealed a high intake of saturated fats. 


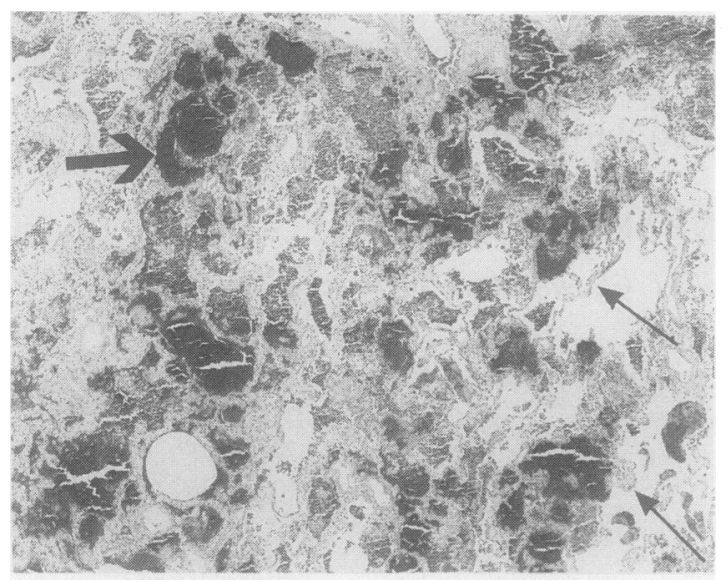

Fig 2 Open lung biopsy specimen stained by haematoxylin and eosin to show widespread intra-alveolar haemorrhage and small foci of desquamation. The broad arrow shows an area of alveolar haemorrhage, also seen elsewhere, and the two thin arrows indicate areas of focal desquamation.

\section{Discussion}

The onset of symtoms two days after the start of treatment with nitrofurantoin, the recovery-albeit slow-after withdrawal of the drug, and the absence of any recurrence or evidence of multiorgan disease all fit the theory that this was a reaction to nitrofurantoin. This case corresponds to the fifth category of causes of alveolar haemorrhage-that due to exogenous agents-described by Leatherman et $^{\mathrm{al}}{ }^{4}$ in a recent comprehensive review. Presumably the slow rate of recovery relates to the severity of the lung damage; other reports, including one of adult respiratory distress syndrome associated with nitrofurantoin, ${ }^{5}$ suggest that four to five days may elapse before clinical or radiographic improvement occurs.
The mechanism of lung reactions to nitrofurantoin is not known; possibly this relates in part to the lack of histological material in reported series. Sasame $e t a^{6}$ suggested that nitrofurantoin stimulated the production of superoxide and hydrogen peroxide in lung microsomes and Boyd $\mathrm{et} \mathrm{al}^{7}$ later $\frac{\bar{\sigma}}{\bar{\omega}}$ showed that vitamin $E$ pretreatment and a diet containing a $\vec{\sigma}$ high proportion of saturated fatty acids protected rats from $\propto$ nitrofurantoin lung damage. Our patient had normal vita- ® min $E$ concentrations and a high normal saturated fat intake. She had no evidence of a generalised clotting. disorder.

We therefore suggest that our patient's reaction to nitro- $\vec{\omega}$ furantoin with histologically confirmed alveolar haemorrhage, desquamation, and a subsequent pleural effusion $x$ represents the severe end of a range of pulmonary reactions $\vec{N}$ to this drug.

\section{References}

1 Israel HL, Diamond P. Recurrent pulmonary infiltration and pleural effusion due to nitrofurantoin sensitivity. $N$ Engl J Med $\overparen{D}$ 1962;266:1024-6.

2 Hailey FJ, Glascock HW jun, Hewitt WF. Pleuropneumonic reactions to nitrofurantoin. $N$ Engl J Med 1969;281:1087-90.

3 Auerbuch SD, Yungbluth P. Fatal pulmonary haemorrhage due to nitrofurantoin. Arch Intern Med 1980;140:271-3.

4 Leatherman JW, Davies SF, Hoidal JR. Alveolar haemorrhage syndromes: diffuse microvascular lung haemorrhage in immune and idiopathic disorders. Medicine (Baltimore) 1984;63:343-61.

5 Israel RH, Gross RA, Bomba P. Adult respiratory distress syndrome associated with acute nitrofurantoin toxicity. Respiration 1980;39:318-22.

6 Sasame HA, Boyd MR. Superoxide and hydrogen peroxide production and NADPH oxidation stimulated by nitrofurantoin in lung microsomes: possible implications to toxicity. Life Sci 1979;24:1091-6.

7 Boyd MR, Catignani GL, Sasame HA, et al. Acute pulmonary injury in rats by nitrofurantoin and modification by vitamin $\mathrm{E}$, dietary fat and oxygen. Am Rev Respir Dis 1979;120:93-9. 